\title{
Arbitrage-Free Conditions and Hedging Strategies for Markets with Penalty Costs on Short Positions
}

\author{
O. L. V. Costa and E. V. Queiroz Filho \\ Departamento de Engenharia de Telecomunicações e Controle, \\ Escola Politécnica da Universidade de São Paulo, 05508-900 São Paulo, SP, Brazil \\ Correspondence should be addressed to O. L. V. Costa, oswaldo@lac.usp.br
}

Received 25 October 2011; Accepted 13 December 2011

Academic Editor: Weihai Zhang

Copyright (C) 2012 O. L. V. Costa and E. V. Queiroz Filho. This is an open access article distributed under the Creative Commons Attribution License, which permits unrestricted use, distribution, and reproduction in any medium, provided the original work is properly cited.

\begin{abstract}
We consider a discrete-time financial model in a general sample space with penalty costs on short positions. We consider a friction market closely related to the standard one except that withdrawals from the portfolio value proportional to short positions are made. We provide necessary and sufficient conditions for the nonexistence of arbitrages in this situation and for a self-financing strategy to replicate a contingent claim. For the finite-sample space case, this result leads to an explicit and constructive procedure for obtaining perfect hedging strategies.
\end{abstract}

\section{Introduction}

In recent years, applications of stochastic analysis and control have entered in the field of financial engineering in an effective and rapid way, due mainly to the powerful tools that can be brought from these disciplines into almost all aspects of fields like, for instance, in the study of arbitrage, hedging, pricing, and portfolio optimization. One of the classical problems in portfolio optimization is the mean-variance portfolio selection problem, which was transformed with the seminal work of Markowitz in [1] (see also [2]). Since then, the amount of research on this subject has increased in order to provide the development of sophisticated analytical and numerical methods for financial engineering models with more realistic assumptions see, for instance, [3-8], among others. More recently, the multiperiod meanvariance problem was tackled by [9] and later extended in several directions see, for instance, [10-25]. The pioneering work of Black and Scholes [26] and Merton [27] provided a major change in the area of pricing of derivative securities, showing that the analysis should be based on nonarbitrage considerations rather than on preference-related concepts such as 
expected values. From the works of Harrison and Kreps [28] and Harrison and Pliska [29], it became apparent that semimartingale theory provided a natural framework for the analysis of financial markets and pricing.

Another fundamental result on the study of arbitrage, hedging, and pricing of financial markets is the Dalang-Morton-Willinger theorem, also known as the fundamental theorem of asset pricing. It states that in a frictionless security market, the existence of an equivalent martingale measure for the discounted price process is equivalent to the absence of arbitrage (see, e.g., [30]). Recently, there has been a number of papers dealing with contingent claim valuation and extending versions of the aforementioned theorem in several directions (see, e.g., [31-38]). The subject of pricing derivatives with transaction costs and portfolio selection with transaction costs is of practical importance and has been in evidence over the last years. Two types of transaction costs are considered; fixed costs, which are paid whenever there is a change of position, and proportional costs, which are charged according to the volume traded. Different approaches to the problem of pricing derivatives with transaction costs and the portfolio choice problem under transaction costs can be found in the literature see, for instance, $[32,37,39-44]$. The results in [45] provided a version of the fundamental theorem of asset pricing within a short sales constraints framework and possible infinite number of transactions within a finite period of time, using the free-lunch notion, a stronger notion of the no arbitrage condition. The case of closed cone constraints on the amount invested in the risky assets, which includes restrictions on short sales, has been studied in [46] for the case in which the price process is positive and under a nondegeneracy hypothesis on the price process. In [47], these results were generalized, and the fundamental theorem of asset pricing was stated under polyhedral convex cone constraints and using the classical notion of no arbitrage instead of free lunch. The general short sales constraints in $[45,47]$ were considered by separating the price process into two sort of securities; those which cannot be held in negative amounts and those that can only be held in negative amounts. The no arbitrage condition in this case is shown in [47] to be equivalent to the existence of a positive interest rate process and an equivalent probability measure $\mathbf{Q}$ under which the discounted price processes of securities that cannot be sold short are supermartingales, and the discounted price processes of securities that can only be sold short are submartingales.

In this paper, we consider a model closely related to the standard one (see $[30,48,49])$, except that a withdrawal directly proportional to the amount on short positions is made from the portfolio. As far as the authors are aware of, this model has not been studied before (see also Remark 3.3). Theorem 3.1 provides necessary and sufficient conditions for the nonexistence of arbitrages directly in terms of the price process and penalty costs at time $t$ and can be seen as a natural extension of the standard fundamental theorem of asset pricing (see, e.g., $[30,48,49])$. When the penalty costs go to zero, our result reduces to that presented in [30]. From this result we derive a sufficient condition for nonarbitrage and for a self-financing strategy to consistently replicate a contingent claim (e.g., any other superreplicating selffinancing strategy will have an initial value greater than that of the replicanting strategy). For the finite sample space case, this result yields an explicit and constructive procedure for obtaining perfect hedging strategies.

This paper is organized in the following way. Section 2 presents some notation, definitions, and the financial model. Section 3 contains the main results of the paper. In Section 4, we present an explicit and constructive procedure for obtaining perfect hedging strategies for the case in which the sample space is finite, as well as some numerical examples. Section 5 concludes the paper. The proof of some auxiliary results and the main results are presented in the appendix. 


\section{Notation, Definitions and Problem Formulation}

Let the real $d$-dimensional vector space be denoted by $\mathbb{R}^{d}$ and for $x \in \mathbb{R}^{d}$ we will write $x_{i}$ for the $i$ th component of the vector. The superscript will be omitted for the case $d=1$. For $x, y$ in $\mathbb{R}^{d}$, we set

$$
x \cdot y=\sum_{i=1}^{d} x_{i} y_{i}
$$

We write $x \geq 0$ to denote that all components of $x$ are positive, that is, $x_{i} \geq 0$ for $i=1, \ldots, d$. For $x \in \mathbb{R}^{d}$, we set the following vectors in $\mathbb{R}^{d}: x^{+} \geq 0$ such that its $i$ th component $x_{i}^{+}$is equal to $x_{i}$ if $x_{i} \geq 0$, zero otherwise, $x^{-}=(-x)^{+} \geq 0$ (therefore, $x=x^{+}-x^{-}$and $x_{i}^{+} x_{i}^{-}=0$ for each $i=1, \ldots, d)$. The vector formed by 1 in all components will be represented by $e$, and the vector with 1 at the $i$ th component and 0 elsewhere by $b^{i}$. For a real number $a$, we define $a^{\oplus}=1 / a$ if $a \neq 0$, zero otherwise.

Let $(\Omega, \mathcal{F}, \mathbf{P})$ be a complete probability space equipped with a filtration $\left\{\mathcal{F}_{t}\right\}, t=$ $0,1, \ldots, T$. For $\mathcal{G}$ a sub- $\sigma$-algebra of $\mathcal{F}$, we denote by $\mathcal{L}_{0}^{d}(\Omega, \mathcal{G}, \mathbf{P})$ (or simply $\mathcal{L}_{0}^{d}(\mathcal{G})$ ) the space of $\mathcal{G}$-measurable random variables with values in $\mathbb{R}^{d}$, which is a complete topological vector space if equipped with the topology of convergence measure. As any sequence converging in probability contains a subsequence converging almost surely (a.s.), we can assume without loss of generality that any convergent sequence in $\mathcal{L}_{0}^{d}(\mathcal{G})$ will converge a.s. For any probability measure $Q, E_{Q}(\cdot)$ denotes the expectation with respect to $Q$, and we write $\mathbf{Q} \sim \mathbf{P}(\mathbf{Q}<<\mathbf{P})$ whenever the probability measure $\mathbf{Q}$ is equivalent to (absolutely continuous with respect to) $\mathbf{P}$. For any $A \in \mathcal{F}, 1_{A}$ denotes the indicator function of the set $A$. Let $\mathcal{L}_{0}^{d+}(\mathcal{G})$ be the set of random vectors $X \in \mathcal{L}_{0}^{d}(\mathcal{G})$ such that $\mathbf{P}(X \geq 0)=1$. The space of integrable random vectors in $\mathcal{L}_{0}^{d}(\mathcal{G})$ will be denoted by $\mathcal{L}_{1}^{d}(\mathcal{G})$ and the space of essentially bounded random vectors in $\mathcal{L}_{0}^{d}(\mathcal{G})$ by $\mathcal{L}_{\infty}^{d}(\mathcal{G})$.

Consider given stochastic processes $S=\{S(t) ; t=0, \ldots, T\}$ and $D=\{D(t) ; t=$ $1, \ldots, T\}$ taking values in $\mathbb{R}^{d}$ with $S(t) \in \mathcal{L}_{0}^{d}\left(\Psi_{t}\right)$ and $S_{1}(t)=1$ for each $t=0, \ldots, T$, and $D(t) \in \mathcal{L}_{0}^{d+}\left(\Psi_{t}\right)$ for each $t=1, \ldots, T$. We define for $t=1, \ldots, T, \Delta S(t):=S(t)-S(t-1)$. A trading strategy $H=(H(1), \ldots, H(T))$ is defined such that each $H(t)$ is a $d \times 2$-dimensional random matrix with columns $H^{+}(t) \in \mathcal{L}_{0}^{d+}\left(\mathcal{F}_{t-1}\right)$ and $H^{-}(t) \in \mathcal{L}_{0}^{d+}\left(\mathcal{F}_{t-1}\right)$. It describes an investor's portfolio as carried forward from time $t=0$ to time $t=T$. In the model of a security market, $S$ describes the evolution of the prices of $d$ securities, $H^{+}(t)$ represents the number of units of each security hold in a long position from time $t-1$ to time $t, H^{-}(t)$ represents the number of units of each security hold in a short selling position from time $t-1$ to time $t$, and $D$ the evolution of the penalty costs and possible spread costs between borrowing and lending rates due to short selling positions on each security $i$.

Associated to a trading strategy $H$, we have the value process $V^{H}:=\left(V^{H}(0), \ldots\right.$, $\left.V^{H}(T)\right)$ describing the total value of the portfolio at each time $t$. For notational simplicity, we will omit the superscript $H$ whenever no confusion arises. The portfolio value can be written, at time $t=0$, as

$$
V(0)=\left(H^{+}(1)-H^{-}(1)\right) \cdot S(0),
$$

and at times $t=1, \ldots, T$, as

$$
V(t)=\left(H^{+}(t)-H^{-}(t)\right) \cdot S(t)-H^{-}(t) \cdot D(t) .
$$


The quantity $V(t)$ represents the value of the portfolio at time $t$ just before any change of ownership positions take place at that time. The penalty costs due to short selling positions are represented by the costs:

$$
H^{-}(t) \cdot D(t)
$$

The value of the portfolio at time $t+1$ just after the change of ownership positions is $\left(\mathrm{H}^{+}(t+\right.$ $\left.1)-H^{-}(t+1)\right) \cdot S(t)$. We consider in this paper self-financing trading strategies, so that no money is added or withdrawn from the portfolio between times $t=0$ to time $t=T$. Any change in the portfolio's value is due to a gain or loss in the investments, and penalty costs due to the short selling positions. Thus, we must have

$$
V(t)=\left(H^{+}(t+1)-H^{-}(t+1)\right) \cdot S(t)
$$

From (2.2)-(2.5), we have for $t=1, \ldots, T$ that

$$
V(t)=V(t-1)+\left(H^{+}(t)-H^{-}(t)\right) \cdot \Delta S(t)-H^{-}(t) \cdot D(t) .
$$

We notice that the penalties can be seen as withdrawals from the portfolio value proportional to the short selling positions.

We conclude this section with the definition of an arbitrage opportunity. We say that there is an arbitrage opportunity if for some self-financing trading strategy $H$, we have a.s. that
(i) $V(0) \leq 0$,
(ii) $V(T) \geq 0$, and
(iii) $E(V(T))>0$.

\section{Main Results}

In this section, we present the main results of the paper. We start with Theorem 3.1, which provides necessary and sufficient conditions for the nonexistence of arbitrages and can be seen as a natural extension of the standard fundamental theorem of asset pricing (see, e.g., $[30,48,49])$. As pointed out in Remark 3.2, when the penalty costs go to zero, our result reduces to that presented in [30]. In Remark 3.3, we point out the differences between our result and previous results presented in the literature. As usual in this kind of problems, the hardest part of the proof is to show that a certain set is closed (see Proposition A.1 in the appendix). In the sequence, we derive a sufficient condition for nonarbitrage and for a selffinancing strategy to consistently replicate a contingent claim. In Section 4, we consider the finite sample space case so that the results in this section yield an explicit and constructive procedure for obtaining perfect hedging strategies.

The following theorem provides necessary and sufficient conditions for the nonexistence of arbitrages. The proof can be found in the appendix. In what follows, we recall that $\mathcal{L}_{\infty}^{+}\left(\mathcal{F}_{t}\right)$ represents the space of essentially bounded $\mathscr{F}_{t}$-measurable random variables $Z$ such that $\mathbf{P}(Z \geq 0)=1$. 
Theorem 3.1. The following statements are equivalent:

(i) there are no arbitrage opportunities,

(ii) for any self-financing strategy $H$, one has a.s. that

$$
\left.\begin{array}{c}
\left(H^{+}(t)-H^{-}(t)\right) \cdot S(t-1) \leq 0, \\
\left(H^{+}(t)-H^{-}(t)\right) \cdot \Delta S(t)-H^{-}(t) \cdot D(t) \geq 0
\end{array}\right\} \Longrightarrow\left(H^{+}(t)-H^{-}(t)\right) \cdot \Delta S(t)-H^{-}(t) \cdot D(t)=0,
$$

(iii) there exists a stochastic process $\{r(t)\}$ with $r(t) \in \mathcal{L}_{\infty}^{+}\left(\mathcal{F}_{t}\right)$ for each $t=0,1, \ldots, T-1$ and a probability measure $\mathbf{Q} \sim \mathbf{P}$ such that $0<d \mathbf{Q} / d \mathbf{P} \in \mathcal{L}_{\infty}^{+}(\boldsymbol{F}), S(t), D(t)$ are integrable with respect to $\mathbf{Q}$ and for each $t=0, \ldots, T-1$,

$$
E_{\mathbf{Q}}\left(\Delta S(t+1) \mid \mathcal{F}_{t}\right) \leq r(t) S(t) \leq E_{\mathbf{Q}}\left(\Delta S(t+1)+D(t+1) \mid \mathcal{F}_{t}\right) \text { a.s. }
$$

Remark 3.2. For the case in which $D(t)=0$, our results reduce to the well-known fundamental theorem of asset pricing with finite-discrete time and infinite state space, see [30] (recall that $S_{1}(t)=1, \Delta S_{1}(t)=0$, and if $D_{1}(t)=0$, then (3.2) implies that $\left.r(t)=0\right)$.

Remark 3.3. In $[45,47]$, the authors consider a financial market with two sort of securities, those that cannot be held in negative amounts and represented by $\bar{S}(t)$, and those that can only be held in negative amounts and represented by $\widetilde{S}(t)$. To write our problem in the above set-up, we would need to define the fictitious price processes $\bar{S}(t), \widetilde{S}(t)$ as: $\bar{S}(0)=\widetilde{S}(0)=S(0)$ and for $t=1, \ldots, T, \bar{S}(t)=S(t), \widetilde{S}(t)=S(t)+D(1)+\cdots+D(t)$. Notice that there is no discounting to be applied since we are considering that $S_{1}(t)=1$. By doing this, we would have that for $t=1, \ldots, T, \Delta \bar{S}(t)=\Delta S(t), \Delta \widetilde{S}(t)=\Delta S(t)+D(t)$ and this would yield that $V(t)=V(t-1)+\left(H^{+}(t)-H^{-}(t)\right) \cdot \Delta S(t)-H^{-}(t) \cdot D(t)$, which is similar to (2.6). Note, however, that the models are different since we cannot guarantee that a self-financing strategy $H$ for the above model will be self-financing for our model, and vice versa. Indeed, for the above model, the self-financing condition would read as $\left(H^{+}(t+1)-H^{-}(t+1)\right) \cdot S(t)-H^{-}(t+1) \cdot \sum_{k=1}^{t} D(k)=$ $\left(H^{+}(t)-H^{-}(t)\right) \cdot S(t)-H^{-}(t) \cdot \sum_{k=1}^{t} D(k)$, while for our model it would be $\left(H^{+}(t+1)-H^{-}(t+1)\right)$. $S(t)=\left(H^{+}(t)-H^{-}(t)\right) \cdot S(t)-H^{-}(t) \cdot D(t)$. This also occurs with the nonarbitrage conditions of the two models. Indeed, the nonarbitrage condition presented in $[45,47]$ states that $\bar{S}(t)$ is a supermartingale and $\widetilde{S}(t)$ is a submartingale, which would involve the sum of the terms $(k)$. On the other hand, for our model, the nonarbitrage condition (3.2) involves only the state price $S(t), S(t+1)$ and penalty costs $D(t+1)$ emphasizing the difference between the two models.

In what follows, we define

$$
\mathcal{K}=\{(\{r(t)\}, \mathbf{Q}) ; r(t) \text { and } \mathbf{Q} \text { satisfying condition (iii) of Theorem 3.1 }\}
$$

We recall next that a contingent claim (random variable) $X \in \mathcal{L}_{0}\left(\mathcal{F}_{T}\right)$ is marketable if for some self-financing strategy $H$ we have that a.s. $X=V^{H}(T)$ and, in this case, $H$ is said to replicate $X$. We say that $H$ superreplicates $X$ if a.s. we have that $V^{H}(T) \geq X$. We have the following corollary (see the proof in the appendix). 
Corollary 3.4. Suppose that $H$ superreplicates $X$ and there is no arbitrage. Then, for any $(\{r(t)\}$, Q) $\in \mathcal{K}$ one has a.s. that

$$
E_{\mathbf{Q}}\left(\frac{X}{(1+r(T)) \cdots(1+r(t))} \mid \mathscr{F}_{t}\right) \leq V(t) .
$$

Writing $\Gamma(t)=\left\{\omega ; V(t)(\omega)-E_{\mathbf{Q}}\left(X /(1+r(T)) \cdots(1+r(t)) \mid \mathcal{F}_{t}\right)(\omega)>0\right\}$ one has that if $\mathbf{P}(\Gamma(T))>$ 0 then $\mathbf{P}(\Gamma(t))>0$ for every $t=T-1, \ldots, 0$.

We will be interested now in deriving a condition such that the pricing of a marketable contigent claim $X \in \mathcal{L}_{0}\left(\mathscr{F}_{T}\right)$ is obtained from a self-financing strategy $H$ that replicates $X$ with $H^{+}(t) \cdot H^{-}(t)=0$ for $t=1, \ldots, T$, so that logical pricing can be obtained in this way. Let us define $\mathbb{J}:=\left\{a=\{a(t)\}_{t=0}^{T-1}\right.$; for $t=0, \ldots, T-1, a(t) \in \mathcal{L}_{0}^{d+}\left(\mathcal{F}_{t}\right), a_{i}(t)=0$ or 1$\}$. For a self-financing strategy $H$ satisfying $H^{+}(t) \cdot H^{-}(t)=0$, we set $a^{H}=\left\{a^{H}(t)\right\} \in \mathbb{J}$ as $a_{i}^{H}(t)=$ $1_{\left\{H_{i}^{-}(t+1)>0\right\}}$.

Definition 3.5. For $a=\{a(t)\} \in \mathbb{J}$, set

$$
\begin{gathered}
\Theta^{a}=\left\{\mathbf{Q} \sim \mathbf{P}, \frac{d \mathbf{Q}}{d \mathbf{P}} \in \mathcal{L}_{\infty}^{+}(\mathcal{F}) ; S(t), D(t) \text { are integrable with respectto } \mathbf{Q},\right. \\
\text { for } t=0, \ldots, T, E_{\mathbf{Q}}\left(\Delta S_{i}(t+1) \mid \mathcal{F}_{t}\right)=0 \text { a.s. on }\left\{a_{i}(t)=0\right\}, \text { and } \\
\left.E_{\mathbf{Q}}\left(\Delta S_{i}(t+1)+D_{i}(t+1) \mid \mathcal{F}_{t}\right)=0 \text { a.s. on }\left\{a_{i}(t)=1\right\}\right\} .
\end{gathered}
$$

We have the following proposition showing that any $a=\{a(t)\} \in \mathbb{J}$ will lead to an element in $\mathcal{K}$ (see the proof in the appendix).

Proposition 3.6. If for some $a=\{a(t)\} \in \mathbb{J}$ one has $\Theta^{a} \neq \emptyset$, then there are no arbitrages.

Finally, we have the following result, presenting a sufficient condition for a self-financing strategy to consistently replicate a contingent claim (i.e., any other superreplicating self-financing strategy will have an initial value greater than that of the replicating strategy). The proof can be found in the appendix.

Proposition 3.7. If $H$ is a self-financing strategy that replicates $X$ with $H^{+}(t) \cdot H^{-}(t)=0$ for $t=1$, $\ldots, T$ and $\Theta^{a^{H}} \neq \emptyset$, then for any superreplicating strategy $\widehat{H}$ for $X$ one has a.s. for $t=0,1, \ldots, T$ that $V^{\widehat{H}}(t) \geq V^{H}(t)$ and if $\mathbf{P}\left(V^{\widehat{H}}(T)>X\right)>0$, then $\left(V^{\widehat{H}}(t)>V^{H}(t)\right)>0$.

\section{A Numerical Procedure}

In this section, we consider the finite-state space case and present an algorithm for obtaining the hedging strategy for a marketable claim $X$ satisfying the conditions of Proposition 3.7. We assume here that $\Omega=\left\{\omega_{1}, \ldots, \omega_{\kappa}\right\}$ and that $D(t)$ is $\mp_{t-1}$-measurable. We consider the single period case only, and suppress the time dependence whenever it is possible. The multiperiod case follows in a similar way, by using the information structure described in [50] or [51], and 
by applying backwards in time, the procedure described here for the single period case and each node of the information structure. Define the following matrix $A$ : $A=\left(A_{1} A_{2}\right)$, where

$$
\mathrm{A}_{1}=\left(\begin{array}{cccc}
1 & S_{2}(1)\left(\omega_{1}\right) & \ldots & S_{d}(1)\left(\omega_{1}\right) \\
\vdots & \vdots & \ddots & \vdots \\
1 & S_{2}(1)\left(\omega_{\kappa}\right) & \ldots & S_{d}(1)\left(\omega_{\kappa}\right)
\end{array}\right), \quad A_{2}=-\left(A_{1}+D\right)
$$

with $D=e \mathbf{d}$ (recall that $e$ is the vector formed by 1 in all components), $\mathbf{d}=\left(g_{1} \ldots g_{d}\right)$. Let the vector $x \in \mathbb{R}^{\kappa}$ be such that $x_{j}=X\left(\omega_{j}\right), j=1, \ldots, \kappa$. We have that $X$ is marketable if and only if there exists $H^{+}, H^{-}$that satisfy the system:

$$
\begin{gathered}
A\left(\begin{array}{c}
H^{+} \\
H^{-}
\end{array}\right)=A_{1}\left(H^{+}-H^{-}\right)-D H^{-}=x, \\
H^{+} \geq 0, \quad H^{-} \geq 0 .
\end{gathered}
$$

For the case in which $\kappa=d$ and $A_{1}$ has an inverse, we have the following explicit and constructive procedure for obtaining a trading strategy $H$ that replicates $X$ with $H^{+} \cdot H^{-}=0$. Since $D=e \mathrm{~d}$ and $A_{1}^{-1} e=b^{1}$ (recall that $b^{1}$ is the vector formed by 1 at the 1 st component, and 0 elsewhere), we have premultiplying (4.2) by $A_{1}^{-1}$ that $H^{+}, H^{-}$satisfy (4.2) if and only if satisfy

$$
\left(H^{+}-H^{-}\right)-A_{1}^{-1} e \mathbf{d} H^{-}=\left(H^{+}-H^{-}\right)-b^{1} \mathbf{d} H^{-}=A_{1}^{-1} x .
$$

Set $y=A_{1}^{-1} x$. Let us obtain $H$ that satisfies (4.2), (4.3) with $H^{+} \cdot H^{-}=0$. Define for $i=2$, $\ldots, d: H_{i}^{+}=y_{i}, H_{i}^{-}=0$ if $y_{i} \geq 0$, otherwise, $H_{i}^{-}=-y_{i}, H_{i}^{+}=0$. For $i=1$, calculate

$$
z=y_{1}+\sum_{i=2}^{d} g_{i} H_{i}^{-} \text {. }
$$

In order to have (4.4) satisfied, we must have $\left(H_{1}^{+}-H_{1}^{-}\right)-g_{1} H_{1}^{-}=z$. If $z \geq 0$, then set $H_{1}^{+}=z$, $H_{1}^{-}=0$, otherwise, set $H_{1}^{+}=0$ and $H_{1}^{-}=-z /\left(1+g_{1}\right)$. Thus, we have obtained in this way a trading strategy $H$ that replicates $X$ with $H^{+} \cdot H^{-}=0$.

Finally, notice that for the discrete sample space, the set $\mathbb{J}$ is finite, and thus if we assume that for every $a \in \mathbb{J}, \Theta^{a} \neq \emptyset$, then the conditions of Proposition 3.7 will be satisfied. With the above procedure, we have a seller price and a buyer price for each contingent claim. The seller price, denoted by $V_{s}(0)$, is obtained by applying to $X$ backwards in time the algorithm presented above. The buyer price, denoted by $V_{b}(0)$, is obtained by applying the backward algorithm to $-X$, and taking $V_{b}(0)=-V(0)$. We illustrate this procedure next for the binomial case.

Example 4.1. Let us consider the binomial model, which consists of a single risky security satisfying

$$
S_{2}(t)=\frac{1}{B(t)} u^{N(t)} d^{t-N(t)} S_{2}(0),
$$


$t=1, \ldots, T$, where $0<d<1<u$ and $N=\{N(t) ; t=1, \ldots, T\}$ is a binomial process with parameter $p, 0<p<1$, and the bank account is given by $B(t)=\left(1+r_{f}\right)^{t}, t=0,1, \ldots, T$. The penalty costs are assumed to be of the form:

$$
D_{1}(t)=\alpha_{1} \frac{1}{1+r_{f}}, \quad D_{2}(t)=\alpha_{2} \frac{S_{2}(t-1)}{1+r_{f}} .
$$

It is easy to see that in this case $\mathbb{J}=\{(0,0),(0,1),(1,0),(1,1)\}$, and we have the following possibilities for $\Theta^{a}=\left\{\pi_{1}, \pi_{2}\right\}$, where $\pi_{1}$ is associated to the probability measure $\mathbf{Q}$ when the stocks goes up, $\pi_{2}$ when the stocks goes down:

(i) $a=(0,0)$; in this case,

$$
\pi_{1}=\frac{1+r_{f}-d}{u-d}, \quad \pi_{2}=\frac{u-\left(1+r_{f}\right)}{u-d} ;
$$

(ii) $a=(1,0)$; in this case,

$$
\pi_{1}=\frac{1+r_{f}-\alpha_{1}-d}{u-d}, \quad \pi_{2}=\frac{u-\left(1+r_{f}-\alpha_{1}\right)}{u-d}
$$

(iii) $a=(0,1)$; in this case,

$$
\pi_{1}=\frac{1+r_{f}+\alpha_{0}-d}{u-d}, \quad \pi_{2}=\frac{u-\left(1+r_{f}+\alpha_{0}\right)}{u-d}
$$

(iv) $a=(1,1)$; in this case,

$$
\pi_{1}=\frac{1+r_{f}+\alpha_{0}-\alpha_{1}-d}{u-d}, \quad \pi_{2}=\frac{u-\left(1+r_{f}+\alpha_{0}-\alpha_{1}\right)}{u-d} .
$$

From above, it is clear that the condition which guarantees that $\Theta^{a} \neq \emptyset$, and thus that the conditions of Proposition 3.7, will be satisfied, is given by $u>1+r_{f}+\alpha_{1}$ and $d<1+r_{f}-\alpha_{2}$.

Let us consider the following numerical example. Suppose that $S_{2}(0)=5, u=4 / 3$, $d=8 / 9, \alpha_{1}=\alpha_{2}=1 / 30, r_{f}=1 / 9$. For this case, we have $1+r_{f}+\alpha_{1}=103 / 90<u=4 / 3$, and $1+r_{f}-\alpha_{2}=97 / 90>d=8 / 9$, and the conditions of Proposition 3.7 will be verified. Let us consider the following option: $X=\max \{S(2)-5,0\}$. By applying the backward procedure described above, we obtain that the seller price for $X$ is $V_{s}(0)=1.3272$, with the following hedging strategy: $H_{1}^{+}(0)=0, H_{1}^{-}(0)=2.796, H_{2}^{+}(0)=0.8246, H_{2}^{-}(0)=0$, and for the case in which the risky security goes up, $H_{1}^{+}(1)=0, H_{2}^{-}(1)=3.932, H_{2}^{+}(1)=1.0, H_{2}^{-}(1)=0, V(1)=$ 2.2977, while for the case in which it goes down, $H_{1}^{+}(0)=0, H_{1}^{-}(0)=1.4563, H_{2}^{+}(0)=0.4687$, $H_{2}^{-}(0)=0$, and $V(1)=0.4652$.

By repeating the procedure now for $-X$, we obtain that the buyer price for $X$ is $V_{b}(0)=$ 0.9355, with the following hedging strategy: $H_{1}^{+}(0)=2.6926, H_{1}^{-}(0)=0, H_{2}^{+}(0)=0, H_{2}^{-}(0)=$ 0.7256 , and for the case in which the risky security goes up, $H_{1}^{+}(1)=4.23, H_{1}^{-}(1)=0, H_{2}^{+}(1)=$ $0, H_{2}^{-}(1)=1.0, V(1)=1.9667$, while for the case in which it goes down, $H_{1}^{+}(0)=1.5562$, 
$H_{1}^{-}(0)=0, H_{2}^{+}(0)=0, H_{2}^{-}(0)=0.4688$, and $V(1)=0.3542$. As expected, $V_{b}(0)=0.9355<$ $V_{s}(0)=1.3272$.

\section{Conclusions}

In this paper, we study a discrete time with infinite sample space financial model with penalty costs on short selling positions. Unlike previous works, we consider only one price structure for both short and long positions, with the penalties being withdrawals from the portfolio proportional to the short selling position. Our main result, Theorem 3.1, provides necessary and sufficient conditions for the nonexistence of arbitrages and can be seen as an extension of the standard fundamental theorem of asset pricing. When the penalty costs go to zero our result reduces to that presented in [30]. We also present a sufficient condition for a selffinancing strategy to consistently replicate a contingent claim. For the finite-sample space case, this result leads to an explicit and constructive procedure for obtaining perfect hedging strategies. Some examples are presented to illustrate the possible applications of the model.

\section{Appendices}

We present in this appendix the proof of the main results in Section 3. First, we need some auxiliary results, presented next. In what follows, we recall that $\mathcal{L}_{0}^{d}(\mathcal{G})$ represents the space of $\mathcal{G}$-measurable random vectors with values in $\mathbb{R}^{d}, \mathcal{L}_{0}^{d+}(\mathcal{G}) \subset \mathcal{L}_{0}^{+}(\mathcal{G})$, the space of $\mathcal{G}$-measurable random vectors $Z$ such that $\mathbf{P}(Z \geq 0)$ and, for simplicity, $\mathcal{L}_{0}(\mathcal{G})=\mathcal{L}_{0}^{1}(\mathcal{G}), \mathcal{L}_{0}^{+}(\mathcal{G})=\mathcal{L}_{0}^{1+}(\mathcal{G})$. The definition for $\mathcal{L}_{0}^{d}(\mathcal{F}), \mathcal{L}_{0}^{d+}(\mathcal{F})$ and $\mathcal{L}_{0}(\mathcal{F}), \mathcal{L}_{0}^{+}(\mathcal{F})$ is similar.

\section{A. Some Auxiliary Results}

Let $Y \in \mathcal{L}_{0}^{d}(\mathcal{G}), \in \mathcal{L}_{0}^{d}(\mathcal{F})$, and $D \in \mathcal{L}_{0}^{d+}(\mathcal{F})$. We set

$$
\begin{aligned}
K_{Y, X} & =\left\{(\alpha \cdot Y, \alpha \cdot X) ; \alpha \in \mathcal{L}_{0}^{d+}(\mathcal{G})\right\}, \\
N_{Y, X, D} & =\left\{(\beta \cdot Y, \beta \cdot(X+D)) ; \beta \in \mathcal{L}_{0}^{d+}(\mathcal{G})\right\}, \\
J_{Y, X, D} & =K_{Y, X}-N_{Y, X, D}, \\
A_{Y, X, D} & =J_{Y, X, D}-\left\{\{0\} \times \mathcal{L}_{0}^{+}(\mathcal{F})\right\} .
\end{aligned}
$$

The following propositions will be crucial for the developing of our results and are based on the arguments presented in [47, 48, 52].

Proposition A.1. The following statements are equivalent:

(i) $A_{Y, X, D} \cap\left\{\{0\} \times \mathcal{L}_{0}^{+}(\mathscr{F})\right\}=(0,0)$,

(ii) $A_{Y, X, D} \cap\left\{\{0\} \times \mathcal{L}_{0}^{+}(\mathcal{F})\right\}=(0,0)$ and $A_{Y, X, D}$ is closed. 
Proof. We have to show that (i) implies that $A_{Y, X, D}$ is closed. For this, we consider sequences $\left\{\alpha^{n}\right\},\left\{\beta^{n}\right\}$ in $\mathcal{L}_{0}^{d+}(\mathcal{G}),\left\{\rho^{n}\right\}$ in $\mathcal{L}_{0}^{+}(\mathcal{F})$, and $\chi \in \mathcal{L}_{0}(\mathcal{G}), \psi \in \mathcal{L}_{0}(\mathcal{F})$ such that a.s.,

$$
\begin{gathered}
\lim _{n \rightarrow \infty}\left(\alpha^{n}-\beta^{n}\right) \cdot Y=X, \\
\lim _{n \rightarrow \infty}\left\{\left(\alpha^{n}-\beta^{n}\right) \cdot X-\beta^{n} \cdot D-\rho^{n}\right\}=\psi .
\end{gathered}
$$

If we can find $\tilde{\alpha}$ and $\tilde{\beta}$ in $\mathcal{L}_{0}^{d+}(\mathcal{G})$ such that $\mathbf{P}$-a.s.

$$
\begin{gathered}
(\tilde{\alpha}-\tilde{\beta}) \cdot Y=X, \\
(\tilde{\alpha}-\tilde{\beta}) \cdot X-\tilde{\beta} \cdot D \geq \psi,
\end{gathered}
$$

then the result is proved since in this case, setting $\rho=(\widetilde{\alpha}-\tilde{\beta}) \cdot X-\tilde{\beta} \cdot D-\psi$, we have from (A.7) that $\rho \in \mathcal{L}_{0}^{+}(\mathcal{F})$ and $\psi=(\tilde{\alpha}-\tilde{\beta}) \cdot X-\tilde{\beta} \cdot D-\rho$, thus $(X, \psi) \in A_{Y, X, D}$. We set $\Omega_{0} \in \mathcal{F}$ such that the limits (A.5) hold and $\left\{\alpha^{n}\right\},\left\{\beta^{n}\right\},\left\{\rho^{n}\right\}, D$ are always nonnegative. It is easy to see that $\left(\Omega_{0}\right)=1$.

We define next $\bar{\alpha}^{n}=\left(\alpha^{n}-\beta^{n}\right)^{+}$and $\bar{\beta}^{n}=\left(\alpha^{n}-\beta^{n}\right)^{-}$so that $\bar{\alpha}^{n}-\bar{\beta}^{n}=\alpha^{n}-\beta^{n}, \bar{\alpha}_{i}^{n} \bar{\beta}_{i}^{n}=0$, $i=1, \ldots, d,\left\|\bar{\alpha}^{n}-\bar{\beta}^{n}\right\|^{2}=\left\|\bar{\alpha}^{n}\right\|^{2}+\left\|\bar{\beta}^{n}\right\|^{2}$, and on $\Omega_{0}$ that

$$
\begin{gathered}
0 \leq \bar{\alpha}^{n} \leq \alpha^{n}, \quad 0 \leq \bar{\beta}^{n} \leq \beta^{n}, \\
\left(\bar{\alpha}^{n}-\bar{\beta}^{n}\right) \cdot X-\bar{\beta}^{n} \cdot D \geq\left(\alpha^{n}-\beta^{n}\right) \cdot X-\beta^{n} \cdot D .
\end{gathered}
$$

Set $\varsigma=\liminf _{n \rightarrow \infty}\left\|\alpha^{n}-\beta^{n}\right\|=\liminf _{n \rightarrow \infty}\left(\left\|\bar{\alpha}^{n}\right\|^{2}+\left\|\bar{\beta}^{n}\right\|^{2}\right)^{1 / 2}$ and $\Omega_{1}=\left\{\omega \in \Omega_{0} ; \varsigma(\omega)<\right.$ $\infty$ \}. From Lemma 2 of [52], we can find subsequences $\left\{\tilde{\alpha}^{k}\right\},\left\{\tilde{\beta}^{k}\right\}$ of, respectively, $\left\{\alpha^{n}\right\},\left\{\beta^{n}\right\}$ such that on $\Omega_{1}, \lim _{k \rightarrow \infty} \widetilde{\alpha}^{k}=\tilde{\alpha}$ and $\lim _{k \rightarrow \infty} \widetilde{\beta}^{k}=\tilde{\beta}$ for some $\tilde{\alpha}, \tilde{\beta}$ in $\mathcal{L}_{0}^{d+}(\mathcal{G})$. Set $\left\{\tilde{\rho}^{k}\right\}$ the corresponding subsequence of $\left\{\rho^{n}\right\}$. It follows that on $\Omega_{1}, \lim _{k \rightarrow \infty}\left(\tilde{\alpha}^{k}-\tilde{\beta}^{k}\right) \cdot Y=(\tilde{\alpha}-\tilde{\beta}) \cdot Y=X$ from (A.5), and (20),

$$
\begin{aligned}
(\tilde{\alpha}-\tilde{\beta}) \cdot X-\tilde{\beta} \cdot D & =\lim _{k \rightarrow \infty}\left\{\left(\tilde{\alpha}^{k}-\tilde{\beta}^{k}\right) \cdot X-\tilde{\beta}^{k} \cdot D\right\} \\
& \geq \liminf _{k \rightarrow \infty}\left\{\left(\tilde{\alpha}^{k}-\tilde{\beta}^{k}\right) \cdot X-\tilde{\beta}^{k} \cdot D-\tilde{\rho}^{k}\right\} \geq \psi .
\end{aligned}
$$

If $\mathbf{P}\left(\Omega_{1}\right)=1$, then from (A.6) and (A.7) the result is proved. Otherwise, we define $\Omega_{2}=\{\omega \in$ $\left.\Omega_{0} ; \varsigma(\omega)=\infty\right\}$. As in $[47,48]$, we form partitions of $\Omega$, and argue on each separate partition as an autonomous space, considering the appropriate restrictions of the random vectors and traces of the $\sigma$-algebras. 
On $\Omega_{2}$, we define $g^{n}=\left\|\bar{\alpha}^{n}-\bar{\beta}^{n}\right\|^{\oplus} \bar{\alpha}^{n}, f^{n}=\left\|\bar{\alpha}^{n}-\bar{\beta}^{n}\right\|^{\oplus} \bar{\beta}^{n}$, and $v^{n}=\left\|\bar{\alpha}^{n}-\bar{\beta}^{n}\right\|^{\oplus} \rho^{n}$. From (A.5), (A.8), it follows that on $\Omega_{2}$,

$$
\begin{aligned}
& \lim _{n \rightarrow \infty}\left(g^{n}-f^{n}\right) \cdot Y=0, \\
& \liminf _{n \rightarrow \infty}\left\{\left(g^{n}-f^{n}\right) \cdot X-f^{n} \cdot D-v^{n}\right\} \geq \lim _{n \rightarrow \infty}\left\|\alpha^{n}-\beta^{n}\right\|^{\oplus}\left\{\left(\alpha^{n}-\beta^{n}\right) \cdot X-\beta^{n} \cdot D-\rho^{n}\right\}=0 .
\end{aligned}
$$

Since on $\Omega_{2},\left\|g^{n}\right\| \leq 1$ and $\left\|f^{n}\right\| \leq 1$, we have again from Lemma 2 of [52] that we can find convergent subsequences $\left\{\tilde{g}^{k}\right\},\left\{\tilde{f}^{k}\right\}$ of, respectively, $\left\{g^{n}\right\},\left\{f^{n}\right\}$, with limits, respectively, $\tilde{g} \geq 0$ and $\tilde{f} \geq 0$. We denote by $\left\{\tilde{\alpha}^{k}\right\},\left\{\tilde{\beta}^{k}\right\},\left\{\tilde{\rho}^{k}\right\}$ the corresponding subsequences of $\left\{\bar{\alpha}^{n}\right\},\left\{\bar{\beta}^{n}\right\}$, $\left\{\rho^{n}\right\}$, obtained from the association with $\left\{g^{n}\right\}$ and $\left\{f^{n}\right\}$. Since for each $i=1, \ldots, d, g_{i}^{n} f_{i}^{n}=0$, it follows that for each $i=1, \ldots, d$,

$$
\tilde{g}_{i} \tilde{f}_{i}=0
$$

From (A.10) we have, that on $\Omega_{2}$,

$$
\begin{aligned}
(\tilde{g}-\tilde{f}) \cdot Y & =\lim _{k \rightarrow \infty}\left(\tilde{g}^{k}-\tilde{f}^{k}\right) \cdot Y=0, \\
(\tilde{g}-\tilde{f}) \cdot X-\tilde{f} \cdot D & =\lim _{k \rightarrow \infty}\left\{\left(\tilde{g}^{k}-\tilde{f}^{k}\right) \cdot X-\tilde{f}^{k} \cdot D\right\} \geq 0,
\end{aligned}
$$

and from (i), it follows that (A.12) and (A.13) imply that

$$
(\tilde{g}-\tilde{f}) \cdot X-\tilde{f} \cdot D=0
$$

We also have on $\Omega_{2}$ that

$$
1=\|\tilde{g}-\tilde{f}\|^{2}=\|\tilde{g}\|^{2}+\|\tilde{f}\|^{2}
$$

We proceed now by applying induction on $d$. Suppose first that $d=1$. We can find a partition of $\Omega_{2}$ into 2 disjoint sets, defined by $\Omega_{2}^{\tilde{g}}=\left\{\omega \in \Omega_{2} ; \tilde{g}(\omega)>0\right\}$, and $\Omega_{2}^{\tilde{f}}=\left\{\omega \in \Omega_{2} ; \tilde{f}(\omega)>0\right\}$. From (A.11) and (A.15) we have that indeed $\Omega_{2}^{\widetilde{g}}$ and $\Omega_{2}^{\tilde{f}}$ form a disjoint partition of $\Omega_{2}$. From (A.12) and (A.14) we have that on $\Omega_{2}^{\tilde{g}}$ (recalling that in this case $\left.\tilde{f}=0\right), Y=0$ and $X=0$, and that $\lim _{k \rightarrow \infty} \tilde{\beta}^{k}=0$ (since $\tilde{\alpha}^{k} \tilde{\beta}^{k}=0$ and $\tilde{g}>0$ ). This implies that on $\Omega_{2}^{\tilde{g}}, \lim _{k \rightarrow \infty}\left(\tilde{\alpha}^{k}-\tilde{\beta}^{k}\right) Y=0$ and as in (A.9), $0=\lim _{k \rightarrow \infty}\left(\left(\widetilde{\alpha}^{k}-\tilde{\beta}^{k}\right) X-\widetilde{\beta}^{k} D\right) \geq \liminf _{k \rightarrow \infty}\left(\left(\tilde{\alpha}^{k}-\tilde{\beta}^{k}\right) X-\widetilde{\beta}^{k} D-\tilde{\rho}^{k}\right) \geq \psi$, and (A.6), (A.7) hold with $\tilde{\alpha}=0, \tilde{\beta}=0$. Similarly, on $\Omega_{2}^{\tilde{f}}, Y=0, X+D=0$, and $\lim _{k \rightarrow \infty} \tilde{\alpha}^{k}=0$, so that again (A.6), (A.7) hold with $\tilde{\alpha}=0, \tilde{\beta}=0$. This completes the proof for $d=1$. 
Suppose now that the equivalence between (i) and (ii) holds for $d-1$, and that (i) holds for. Define the $2 d$ disjoint sets

$$
\begin{aligned}
& \Omega_{2 i}^{\tilde{g}}=\left\{\omega \in \Omega_{2} ; \tilde{g}_{j}(\omega)=0, \tilde{f}_{j}(\omega)=0, j \leq i-1, \tilde{g}_{i}(\omega)>0\right\}, \\
& \Omega_{2 i}^{\tilde{f}}=\left\{\omega \in \Omega_{2} ; \tilde{g}_{j}(\omega)=0, \tilde{f}_{j}(\omega)=0, j \leq i-1, \tilde{g}_{i}(\omega)=0, \tilde{f}_{i}(\omega)>0\right\} .
\end{aligned}
$$

From (A.11) and (A.15), we have that indeed $\Omega_{2 i}^{\tilde{g}}$ and $\Omega_{2 i}^{\tilde{f}}, i=1, \ldots, d$, form a disjoint partition of $\Omega_{2}$. For $i$ fixed, we will consider first a disjoint partition of $\Omega_{2 i}^{\tilde{g}}$. Consider all subsets, indexed by $s$, formed as $v_{s}^{i} \subseteq\{i+1, \ldots, d\}, u_{s}^{i} \subseteq\{i+1, \ldots, d\}$, with $v_{s}^{i} \cap u_{s}^{i}=\emptyset$. Write $w_{s}^{i}=\{i+1, \ldots, d\}-$ $\left(v_{s}^{i} \cup u_{s}^{i}\right)$, and consider a disjoint partition of $\Omega_{2 i}^{\tilde{g}}$ formed by the sets:

$$
\Omega_{2 i v_{s}^{i} u_{s}^{i}}^{\tilde{g}}=\left\{\omega \in \Omega_{2 i}^{\tilde{g}} ; \tilde{g}_{j}(\omega)>0, j \in v_{s}^{i}, \quad \tilde{f}_{r}(\omega)>0, r \in u_{s}^{i}, \widetilde{g}_{m}(\omega)=0, \tilde{f}_{m}(\omega)=0, m \in w_{s}^{i}\right\}
$$

We fix now $v_{s}^{i}, u_{s}^{i}$ and for notational simplicity, we write $v=v_{s}^{i}, u=u_{s}^{i}, w=w_{s}^{i}, \Omega^{\prime}=\Omega_{2 i v_{s}^{i} u_{s}^{i}}^{\tilde{g}}$ and $\mathcal{F}^{\prime}, \mathcal{G}^{\prime}$, respectively, the corresponding trace of the $\sigma$-algebras $\mathcal{F}, \mathcal{G}$ on $\Omega^{\prime}$. Let us consider that $\mathbf{P}\left(\Omega^{\prime}\right)>0$ (otherwise, it could be discarded) and write $\mathbf{P}^{\prime}(\cdot)=\mathbf{P}(\cdot) / \mathbf{P}\left(\Omega^{\prime}\right)$. On the set $\Omega^{\prime}$, we have from (A.12) and (A.14) that

$$
\begin{aligned}
& Y_{i}=-\frac{1}{\widetilde{g}_{i}}\left\{\sum_{j \in v} \tilde{g}_{j} Y_{j}-\sum_{j \in u} \tilde{f}_{j} Y_{j}\right\}, \\
& X_{i}=-\frac{1}{\widetilde{g}_{i}}\left\{\sum_{j \in v} \tilde{g}_{j} X_{j}-\sum_{j \in u} \tilde{f}_{j}\left(X_{j}+D_{j}\right)\right\} .
\end{aligned}
$$

Set the $d$-1-dimensional random vectors $Y^{\prime}, X^{\prime}, D^{\prime}$ as follows: for $j=1, \ldots, i-1, Y_{j}^{\prime}=Y_{j}$, $X_{j}^{\prime}=X_{j}$ and $D_{j}^{\prime}=D_{j}$, for $j=i+1, \ldots, d, Y_{j-1}^{\prime}=Y_{j}$, and

$$
\begin{aligned}
& X_{j-1}^{\prime}= \begin{cases}X_{j}+D_{j}, & j \in u, \\
X_{j}, & j \notin u, j \geq i+1,\end{cases} \\
& D_{j-1}^{\prime}= \begin{cases}0, & j \in u \cup v, \\
D_{j}, & j \notin u \cup v, j \geq i+1 .\end{cases}
\end{aligned}
$$

For $\ell=1,2, \ldots$, define

$$
\tau^{\ell}=\inf \left\{k ; \tilde{g}_{j}^{k}>\tilde{g}_{j}\left(1-\frac{1}{\ell}\right), \forall j \in v \cup\{i\}, \tilde{f}_{r}^{k}>\tilde{g}_{r}\left(1-\frac{1}{\ell}\right), \forall r \in u\right\}
$$


On $\Omega^{\prime}$, we have that $\tau^{\ell}<\infty, \tilde{f}_{j}^{\tau^{\ell}}=0$ for all $j \in v \cup\{i\}$ and $\tilde{g}_{r}^{\tau^{\ell}}=0$ for all $r \in u$, and consequently $\tilde{\beta}_{j}^{\tau^{\ell}}=0$ for all $j \in v \cup\{i\}$ and $\tilde{\alpha}_{r}^{\tau^{\ell}}=0$ for all $r \in u$. Define $\widehat{\alpha}^{\ell}=\widetilde{\alpha}^{\tau^{\ell}}, \widehat{\beta}^{\ell}=\tilde{\beta}^{\tau^{\ell}}$, and $\hat{\rho}^{\ell}=\tilde{\rho}^{\tau^{\ell}}$. From (A.19), we obtain that

$$
\left(\widehat{\alpha}^{\ell}-\hat{\beta}^{\ell}\right) \cdot X-\hat{\beta}^{\ell} \cdot D=\left(\delta^{\ell}-\varepsilon^{\ell}\right) \cdot X^{\prime}-\varepsilon^{\ell} \cdot D^{\prime},
$$

where for $j=1, \ldots, i-1, \delta_{j}^{\ell}=\widehat{\alpha}_{j}^{\ell}, \varepsilon_{j}^{\ell}=\widehat{\beta}_{j}^{\ell}$, for $j \in w, \delta_{j-1}^{\ell}=\widehat{\alpha}_{j}^{\ell}, \varepsilon_{j-1}^{\ell}=\hat{\beta}_{j}^{\ell}$, for $j \in v$, $\delta_{j-1}^{\ell}=\left(\widehat{\alpha}_{j}^{\ell}-\left(\widetilde{g}_{j} / \widetilde{g}_{i}\right) \hat{\alpha}_{i}^{\ell}\right)^{+}, \varepsilon_{j-1}^{\ell}=\left(\widehat{\alpha}_{j}^{\ell}-\left(\tilde{g}_{j} / \widetilde{g}_{i}\right) \widehat{\alpha}_{i}^{\ell}\right)^{-}$, and for $j \in u, \delta_{j-1}^{\ell}=\left(-\hat{\beta}_{j}^{\ell}+\left(\tilde{f}_{j} / \tilde{g}_{i}\right) \hat{\alpha}_{i}^{\ell}\right)^{+}$, $\varepsilon_{j-1}^{\ell}=\left(-\widehat{\beta}_{j}^{\ell}+\left(\tilde{f}_{j} / \widetilde{g}_{i}\right) \widehat{\alpha}_{i}^{\ell}\right)^{-}$. We notice that $\delta^{\ell}$ and $\varepsilon^{\ell}$ belong to $\mathcal{L}_{0}^{d-1+}\left(\Omega^{\prime}, \mathcal{G}^{\prime}, \mathbf{P}^{\prime}\right)$. Similarly, from (A.18), we have that

$$
\left(\widehat{\alpha}^{\ell}-\hat{\beta}^{\ell}\right) \cdot Y=\left(\delta^{\ell}-\varepsilon^{\ell}\right) \cdot Y^{\prime}
$$

Next, we show that $J_{Y^{\prime}, X^{\prime}, D^{\prime}} \subset J_{Y, X, D}$. For this, we establish a mapping $(\alpha, \beta)=F(\delta, \varepsilon)$ such that $\alpha$ and $\beta$ belong to $\mathcal{L}_{0}^{d+}\left(\Omega^{\prime}, \mathcal{G}^{\prime}, \mathbf{P}^{\prime}\right)$ whenever $\delta$ and $\varepsilon$ belong to $\mathcal{L}_{0}^{d-1+}\left(\Omega^{\prime}, \mathcal{G}^{\prime}, \mathbf{P}^{\prime}\right)$, and that

$$
\begin{aligned}
(\delta-\varepsilon) \cdot Y^{\prime} & =(\alpha-\beta) \cdot Y, \\
(\delta-\varepsilon) \cdot X^{\prime}-\varepsilon \cdot D^{\prime} & =(\alpha-\beta) \cdot X-\beta \cdot D .
\end{aligned}
$$

Indeed, setting $\beta_{i}=0$

$$
\alpha_{i}=\max \left\{0,\left\{\left(\varepsilon_{j-1}-\delta_{j-1}\right) \frac{\tilde{g}_{i}}{\widetilde{g}_{j}} \text { for } j \in v\right\},\left\{\left(\delta_{r-1}-\varepsilon_{r-1}\right) \frac{\tilde{g}_{i}}{\tilde{f}_{r}} \text { for } r \in u\right\}\right\},
$$

and for $j=1, \ldots, i-1, \alpha_{j}=\delta_{j}, \beta_{j}=\varepsilon_{j}$, for $j \in w, \alpha_{j}=\delta_{j-1}, \beta_{j}=\varepsilon_{j-1}$, for $j \in v, \alpha_{j}=$ $\left(\delta_{j-1}-\varepsilon_{j-1}\right)+\left(\widetilde{g}_{j} / \widetilde{g}_{i}\right) \alpha_{i}, \beta_{j}=0$, and for $j \in u, \alpha_{j}=0, \beta_{j}=-\left(\delta_{j-1}-\varepsilon_{j-1}\right)+\left(\tilde{f}_{j} / \tilde{g}_{i}\right) \alpha_{i}$, we get from (A.26) that $\alpha, \beta$ belong to $\mathcal{L}_{0}^{d+}\left(\Omega^{\prime}, \mathcal{G}^{\prime}, \mathbf{P}^{\prime}\right)$ whenever $\delta$ and $\varepsilon$ belong to $\mathcal{L}_{0}^{d-1+}\left(\Omega^{\prime}, \mathcal{C}^{\prime}, \mathbf{P}^{\prime}\right)$, and from (A.18) and (A.19), we get that (A.24) and (A.25) are satisfied, yielding the desired inclusion. From this and (i), we can conclude that $A_{Y^{\prime}, X^{\prime}, D^{\prime}} \cap\left\{\{0\} \times \mathcal{L}_{0}^{+}\left(\Omega^{\prime}, \boldsymbol{F}^{\prime}, \mathbf{P}^{\prime}\right)\right\}=(0,0)$ and by the induction hypothesis, we get that $A_{Y^{\prime}, X^{\prime}, D^{\prime}}$ is closed. Therefore, for some $\widetilde{\delta}$ and $\widetilde{\varepsilon}$ belonging to $\mathcal{L}_{0}^{d-1+}\left(\Omega^{\prime}, \mathcal{G}^{\prime}, \mathbf{P}^{\prime}\right)$, and some $\tilde{p} \in \mathcal{L}_{0}^{+}\left(\Omega^{\prime}, \mathcal{F}^{\prime}, \mathbf{P}^{\prime}\right)$, we have from (A.22) and (A.23), and taking $(\widetilde{\alpha}, \widetilde{\beta})=F(\widetilde{\delta}, \widetilde{\varepsilon})$, that

$$
\begin{aligned}
X & =\lim _{\ell \rightarrow \infty}\left(\hat{\alpha}^{\ell}-\hat{\beta}^{\ell}\right) \cdot Y=\lim _{\ell \rightarrow \infty}\left(\delta^{\ell}-\varepsilon^{\ell}\right) \cdot Y^{\prime}=(\delta-\varepsilon) \cdot Y^{\prime} \\
& =\left(\tilde{\delta}^{\ell}-\widetilde{\varepsilon}^{\ell}\right) \cdot Y^{\prime}=(\tilde{\alpha}-\tilde{\beta}) \cdot Y, \\
\psi & =\lim _{\ell \rightarrow \infty}\left(\left(\hat{\alpha}^{\ell}-\hat{\beta}^{\ell}\right) \cdot X-\widehat{\beta}^{\ell} \cdot D-\hat{\rho}^{\ell}\right) \\
& =\lim _{\ell \rightarrow \infty}\left(\left(\delta^{\ell}-\varepsilon^{\ell}\right) \cdot X^{\prime}-\varepsilon^{\ell} \cdot D^{\prime}-\hat{\rho}^{\ell}\right) \\
& =(\tilde{\delta}-\tilde{\varepsilon}) \cdot X^{\prime}-\tilde{\varepsilon}^{\ell} \cdot D^{\prime}-\tilde{p}=(\tilde{\alpha}-\tilde{\beta}) \cdot X-\tilde{\beta} \cdot D^{\prime}-\tilde{p},
\end{aligned}
$$

showing that (A.6) and (A.7) are satisfied on $\Omega^{\prime}$. 
The proof for the sets $\Omega_{2 i v_{s}^{i} u_{s}^{i}}^{\tilde{f}}$ goes along the same lines, bearing in mind that (A.18) and (A.19) are replaced, respectively, by $Y_{i}=1 / \tilde{f}_{i}\left\{\sum_{j \in v} \tilde{g}_{j} Y_{j}-\sum_{j \in u} \tilde{f}_{j} Y_{j}\right\}$ and $X_{i}+D_{i}=$ $\left(1 / \tilde{f}_{i}\right)\left\{\sum_{j \in v} \tilde{g}_{j} X_{j}-\sum_{j \in u} \tilde{f}_{j}\left(X_{j}+D_{j}\right)\right\}$, and that the mapping $F(\delta, \varepsilon)$ is defined such that instead of (A.26), we take $\alpha_{i}=0$ and $\beta_{i}=\max \left\{0,\left\{\left(\varepsilon_{j-1}-\delta_{j-1}\right)\left(\tilde{f}_{i} / \tilde{g}_{j}\right)\right.\right.$ for $\left.j \in v\right\},\left\{\left(\delta_{r-1}-\varepsilon_{r-1}\right)\left(\tilde{f}_{i} / \tilde{f}_{r}\right)\right.$ for $r \in u\}\}$. This completes the proof of the proposition.

In the next proposition let us consider that $Y \in \mathcal{L}_{0}^{d}(\mathcal{G})$ is such that $Y_{1}=1$ and $X \in$ $\mathcal{L}_{0}^{\mathrm{d}}(\mathcal{F})$ is such that $X_{1}=0$. Again we suppose that $\in \mathcal{L}_{0}^{d+}(\mathcal{F})$.

Proposition A.2. The following statements are equivalent:

(i) for any $\alpha, \beta$ in $\mathcal{L}_{0}^{d+}(\mathcal{G})$, one has a.s. that

$$
\left.\begin{array}{c}
(\alpha-\beta) \cdot Y \leq 0 \\
(\alpha-\beta) \cdot X-\beta \cdot D \geq 0
\end{array}\right\} \Longrightarrow(\alpha-\beta) \cdot X-\beta \cdot D=0
$$

(ii) there exists $r \in \mathcal{L}_{\infty}^{+}(\mathcal{G})$ and a probability measure $\mathbf{Q} \sim \mathbf{P}$ such that $0<R=d \mathbf{Q} / d \mathbf{P} \in$ $\mathcal{L}_{\infty}^{+}(\mathcal{F}), Y, X, D$ are integrable with respect to $\mathbf{Q}$, and

$$
E_{\mathbf{Q}}(X \mid \mathcal{G}) \leq r Y \leq E_{\mathbf{Q}}(X+D \mid \mathcal{G}) \text { a.s. }
$$

Proof. First, we note that (i) is equivalent to $A_{Y, X, D} \cap\left\{\{0\} \times \mathcal{L}_{0}^{+}(\mathcal{F})\right\}=(0,0)$. Indeed, if (i) holds, then clearly $A_{Y, X, D} \cap\left\{\{0\} \times \mathcal{L}_{0}^{+}(\mathcal{F})\right\}=(0,0)$. Conversely, suppose that for some $\alpha, \beta$ in $\mathcal{L}_{0}^{d+}(\mathcal{G})$, $(\alpha-\beta) \cdot Y \leq 0$ and $(\alpha-\beta) \cdot X-\beta \cdot D \geq 0$ a.s. Then, by taking $\widetilde{\alpha}_{1}=\alpha_{1}-(\alpha-\beta) \cdot Y \geq 0, \tilde{\alpha}_{i}=\alpha_{i}$, and recalling that $Y_{1}=1$ and $X_{1}=0$ we get that $(\tilde{\alpha}-\beta) \cdot Y=0$ and $(\tilde{\alpha}-\beta) \cdot X-\beta \cdot D=(\alpha-\beta) \cdot X-\beta \cdot D \geq 0$ a.s., which implies that $(\alpha-\beta) \cdot X-\beta \cdot D=0$ a.s.

Let us show first that (ii) implies (i). Consider $\alpha, \beta$ in $\mathcal{L}_{0}^{d+}(\mathcal{G})$ such that $(\alpha-\beta) \cdot Y=0$ and $(\alpha-\beta) \cdot X-\beta D \geq 0$ a.s. From (A.29), we get that a.s.,

$$
\begin{aligned}
0 & \leq E_{\mathbf{Q}}((\alpha-\beta) \cdot X-\beta \cdot D \mid \mathcal{G})=\alpha \cdot E_{\mathbf{Q}}(X \mid \mathcal{G})-\beta \cdot E_{\mathbf{Q}}((X+D) \mid \mathcal{G}) \\
& \leq r(\alpha-\beta) \cdot Y=0,
\end{aligned}
$$

and thus $E_{\mathbf{Q}}((\alpha-\beta) \cdot X-\beta \cdot D)=0$. From the fact that $\mathbf{Q} \sim \mathbf{P}$, we get that $(\alpha-\beta) \cdot X-\beta \cdot D \geq 0$ a.s., which implies that $(\alpha-\beta) \cdot X-\beta \cdot D=0$ a.s. and again, from $\mathbf{Q} \sim \mathbf{P}$, that $(\alpha-\beta) \cdot X-\beta \cdot D=0$ a.s., showing that (ii) implies (i).

Next, we show that (i) implies (ii). In what follows, we recall that for a complete probability space $\left(\Omega, \mathcal{G}, \mathbf{P}^{\prime}\right)$, we denote by $\mathcal{L}_{1}^{d}\left(\Omega, \mathcal{G}, \mathbf{P}^{\prime}\right)$ the space of integrable $\mathcal{G}$-measurable random variables with values in $\mathbb{R}^{d}$, and $Z \in \mathcal{L}_{1}^{d+}\left(\Omega, \mathcal{G}, \mathbf{P}^{\prime}\right)$ if $Z \in \mathcal{L}_{1}^{d}\left(\Omega, \mathcal{G}, \mathbf{P}^{\prime}\right)$ and $\mathbf{P}^{\prime}(Z \geq 0)=1$. We remind that if $\widetilde{\mathbf{P}}<<\mathbf{P}$ then $\widetilde{\mathbf{P}} \sim \mathbf{P}$ if and only if $d \widetilde{\mathbf{P}} / d \mathbf{P}>0$ a.s., and that for any random variable $\eta$ there exists an equivalent probability measure $\widetilde{\mathbf{P}}$ with bounded density such that $\eta$ is integrable under $\widetilde{\mathbf{P}}$ (see [52]). Consider a change of probability measure $d \mathbf{P}^{\prime}=p d \mathbf{P}$ with $0<p \in \mathcal{L}_{\infty}(\mathcal{F})$ such that $Y \in \mathcal{L}_{1}^{d}\left(\Omega, \mathcal{G}, \mathbf{P}^{\prime}\right), X \in \mathcal{L}_{1}^{d}\left(\Omega, \mathcal{F}, \mathbf{P}^{\prime}\right)$, and $D \in \mathcal{L}_{1}^{d+}\left(\Omega, \mathcal{F}, \mathbf{P}^{\prime}\right)$. From (i) (which is invariant under equivalent change of probability) and Proposition A.1, we get that $V:=A_{Y, X, D} \cap\left(\mathcal{L}_{1}\left(\Omega, \mathcal{G}, \mathbf{P}^{\prime}\right) \times \mathcal{L}_{1}\left(\Omega, \mathcal{F}, \mathbf{P}^{\prime}\right)\right)$ is a closed convex set of $\mathcal{L}_{1}\left(\Omega, \mathcal{G}, \mathbf{P}^{\prime}\right) \times \mathcal{L}_{1}\left(\Omega, \mathcal{F}, \mathbf{P}^{\prime}\right)$, 
and that $V \cap\{0\} \times \mathcal{L}_{1}^{+}\left(\Omega, \mathcal{F}, \mathbf{P}^{\prime}\right)=(0,0)$. Therefore, for any $A \in \mathcal{F}$, with $(A)>0,\left(0,1_{A}\right) \in$ $\{0\} \times \mathcal{L}_{1}^{+}\left(\Omega, \mathcal{F}, \mathbf{P}^{\prime}\right)$, and thus does not belong to the set $V$. By the Hahn-Banach Theorem, $\left(0,1_{A}\right)$ can be strongly separated from $V$ by a nonzero linear continuous functional, so that there exists $r_{A} \in \mathcal{L}_{\infty}(\mathcal{G}), Z_{A} \in \mathcal{L}_{\infty}(\mathcal{F}),\left(r_{A}, Z_{A}\right) \neq(0,0)$, such that

$$
\sup _{(\varphi, \theta) \in V} E^{\prime}\left(r_{A} \varphi+Z_{A} \theta\right)<E^{\prime}\left(Z_{A} 1_{A}\right)
$$

where $E^{\prime}$ denotes the expectation with respect to $\mathbf{P}^{\prime}$. We note that $\varphi \in \mathcal{L}_{1}\left(\Omega, \mathcal{G}, \mathbf{P}^{\prime}\right)$ and $\theta \in$ $\mathcal{L}_{1}\left(\Omega, \mathcal{F}, \mathbf{P}^{\prime}\right)$ are such that $\varphi=(\alpha-\beta) \cdot Y$ and $\theta=(\alpha-\beta) \cdot X-\beta \cdot D-\rho$, with, $\beta \in \mathcal{L}_{0}^{d+}(\mathcal{G})$ and $\rho \in \mathcal{L}_{0}^{+}(\mathcal{G})$. By taking $\varphi=0$ and $\theta=-n 1_{B}$ for any $B \in \mathcal{F}$ and positive integer $n$ (just take $\alpha=\beta=0$ and $\left.\rho=n 1_{B}\right)$, we have from (A.31) that $-n E^{\prime}\left(Z_{A} 1_{B}\right)<E^{\prime}\left(Z_{A} 1_{A}\right)$ which implies that $Z_{A} \geq 0$ a.s. Normalizing, we assume that $Z_{A} \leq 1$. Similarly by taking $\varphi=n 1_{B}$ for any $B \in \mathcal{G}$ and $\theta=0$ (this is possible since $Y_{1}=1$ and $X_{1}=0$, just take $\alpha_{1}=n 1_{B}, \alpha_{i}=0$ for $i=2, \ldots, d$, and $\beta=0, \rho=0)$, we get from the same reasons as before that $-r_{A} \geq 0$. Considering now $\varphi=\alpha \cdot Y$ and $\theta=\alpha \cdot X$ we have from (A.31) and the same arguments as before that for every $\alpha \in \mathcal{L}_{\infty}^{d+}(\mathcal{G}), E^{\prime}\left(\alpha \cdot\left(r_{A} Y+Z_{A} X\right)\right) \leq 0$, which implies that a.s.,

$$
E^{\prime}\left(Z_{A} X \mid G\right) \leq-r_{A} Y
$$

Similarly, considering now $\varphi=-\beta \cdot Y$ and $\theta=-\beta \cdot(X+D)$, we have from (A.31) and the same arguments as before that for every $\beta \in \mathcal{L}_{\infty}^{d+}(\mathcal{G}), E^{\prime}\left(\beta \cdot\left(r_{A} Y+Z_{A}(X+D)\right)\right) \geq 0$, which implies that a.s.

$$
E^{\prime}\left(Z_{A}(X+D) \mid G\right) \geq-r_{A} Y
$$

Consider the family of measures:

$$
Q=\left\{Q_{A} ; d Q_{A}=Z_{A} d \mathbf{P}^{\prime}, \forall A \in \mathcal{F} \text { such that } \mathbf{P}^{\prime}(A)>0\right\} .
$$

Clearly, $Q$ is dominated by $\mathbf{P}^{\prime}$ (i.e., $Q_{A} \ll \mathbf{P}^{\prime}$ for every $A \in \mathcal{F}$ such that $\mathbf{P}^{\prime}(A)>0$ ). From the Halmos-Savage Theorem, $Q$ contains a countable equivalent family $\widetilde{Q}=\left\{Q_{A_{k}} ; k \in \mathbb{I}\right\}$, where $\mathbb{I}$ is a countable set. Define $\Gamma=\left\{\omega ; Z_{A_{k}}(\omega)=0\right.$ for every $\left.k \in \mathbb{I}\right\}$. Since $Q$ and $\tilde{Q}$ are equivalent and $Q_{A_{k}}(\Gamma)=0$ for every $k \in \mathbb{I}$, it follows that $Q_{A}(\Gamma)=0$ for every $A \in \mathbb{F}$ such that $\mathbf{P}^{\prime}(A)>0$. We show next that $\mathbf{P}^{\prime}(\Gamma)=0$. Suppose by contradiction that $\mathbf{P}^{\prime}(\Gamma)>0$, so that $Q_{\Gamma}(\Gamma)=E^{\prime}\left(Z_{\Gamma} 1_{\Gamma}\right)=0$. From (A.31), $\sup _{(\varphi, \theta) \in V} E^{\prime}\left(r_{\Gamma} \varphi+Z_{\Gamma} \theta\right)<0$ which is clearly an absurd (just take $=0, \theta=0)$. This shows that $\mathbf{P}^{\prime}(\Gamma)=0$. Define

$$
\begin{aligned}
& Z=C \sum_{k \in \mathbb{I}} \frac{Z_{A_{k}}}{2^{k}} \in \mathcal{L}_{\infty}^{+}\left(\Omega, \mathcal{F}, \mathbf{P}^{\prime}\right), \\
& r=C \sum_{k \in \mathbb{I}} \frac{\left(-r_{A_{k}}\right)}{2^{k}} \in \mathcal{L}_{\infty}^{+}\left(\Omega, \mathcal{G}, \mathbf{P}^{\prime}\right),
\end{aligned}
$$

where $C=\left(\sum_{k \in \mathbb{I}} E^{\prime}\left(Z_{A_{k}}\right) / 2^{k}\right)^{-1}>0$. Note that $\mathbf{P}^{\prime}(Z>0)=1$ since $\mathbf{P}^{\prime}(\Gamma)=0$, and thus $C$ is well defined. Define the probability measure $\mathbf{Q}$ as $=Z d \mathbf{P}^{\prime}$. It is easy to see that $\mathbf{Q} \sim \mathbf{P}^{\prime}$ 
(since $\mathbf{P}^{\prime}(Z>0)=1$ ). Finally, from (A.32), (A.33), (A.35), and the bounded convergence theorem, we obtain (A.29) with $R=p Z$, completing the proof of the proposition.

\section{B. Proof of the Main Results}

We present next the proof of Theorem 3.1.

Proof. Let us show that (i) implies (ii). The proof of this fact follows from the contrapositive. Suppose we can find $\widehat{H}=\left(\widehat{H}^{+} \widehat{H}^{-}\right)$with $\widehat{H}^{+}, \widehat{H}^{-} \in \mathcal{L}_{0}^{d+}\left(\Psi_{t}\right)$ such that a.s., $\left(\widehat{H}^{+}-\widehat{H}^{-}\right) \cdot S(t)=0$, $\mathcal{R}=\left(\widehat{H}^{+}-\widehat{H}^{-}\right) \cdot \Delta S(t+1)-\widehat{H}^{-} \cdot D(t+1) \geq 0$ and $\mathbf{P}(\Gamma)>0$, where $\Gamma=\{\omega ; \mathcal{R}(\omega)>0\}$. Then, we can define an arbitrage opportunity $H$ for the multiperiod market as follows: $H(s)=0$ for $s \leq t, H(t+1)(\omega)=\widehat{H}$ for $\omega \in \Gamma, H(t+1)(\omega)=0$ for $\omega \notin \Gamma, H_{1}^{+}(s)(\omega)=\mathcal{R}(\omega)$ for $\omega \in \Gamma$ and $s \geq t+2$, and $H_{i}^{+}(s)(\omega)=0, H_{i}^{-}(s)(\omega)=0$ in all the other situations. Then, clearly $V(s)=0$ for $s=0, \ldots, t-1,(t)=0$, and from $(2.5),(2.6), V(s)(\omega)=R(\omega)$ for $s=t+1, \ldots, T, \omega \in \Gamma$, and equal to zero for $\omega \notin \Gamma$, showing the result.

Let us show now that (ii) implies (iii). We will show by backward induction on time $t=T, \ldots, 1$ that we can find random variables $0<\tilde{R}(t) \in \mathcal{L}_{\infty}^{+}\left(\Psi_{t}\right), 0 \leq \tilde{r}(t-1) \in \mathcal{L}_{\infty}^{+}\left(\Psi_{t-1}\right)$, and random vectors $\tilde{X}(t) \in \mathcal{L}_{0}^{d}\left(\Psi_{t}\right), \tilde{D}(t) \in \mathcal{L}_{0}^{d+}\left(\Psi_{t}\right)$ that satisfy $E(\|\tilde{X}(t)\| \tilde{R}(t))<\infty$, $E(\|\widetilde{D}(t)\| \widetilde{R}(t))<\infty, E(\|S(t-1)\| \widetilde{R}(t))<\infty$, and a.s., $E\left(\widetilde{R}(t) \mid \mathcal{F}_{t-1}\right)=1$,

$$
E\left(\tilde{X}(t) \widetilde{R}(t) \mid \Psi_{t-1}\right) \leq \tilde{r}(t-1) S(t-1) \leq E\left(\widetilde{R}(t)(\tilde{X}(t)+\tilde{D}(t)) \mid \Psi_{t-1}\right) .
$$

For $t=T$, the result follows from Proposition A.2 with $\mathcal{F}=\mathcal{F}_{T}, \mathcal{G}=\mathfrak{F}_{T-1}, Y=S(T-1)$, $X=\tilde{X}(T)=\Delta S(T), D=\tilde{D}(T)=D(T)$, and $\widetilde{R}(T)=R, \widetilde{r}(T)=r$. Suppose the result holds for $t+1$. Define $\tilde{X}(t)=\Delta S(t) E\left(\widetilde{R}(T) \cdots \widetilde{R}(t+1) \mid \mathcal{F}_{t}\right)$ and $\widetilde{D}(t)=D(t) E\left(\widetilde{R}(T) \cdots \widetilde{R}(t+1) \mid \mathcal{F}_{t}\right)$. Again, the result follows from Proposition A.2 with $\mathcal{F}=\mathcal{F}_{t}, \mathcal{G}=\mathcal{F}_{t-1}, Y=S(t-1), X=\tilde{X}(t)$, $D=\widetilde{D}(t)$, and $\widetilde{R}(t)=R, \widetilde{r}(t-1)=r$, completing the induction argument. Set $\widetilde{R}(0)=1$, and define for $t=1, \ldots, T,(t-1)=\widetilde{R}(0) \cdots \widetilde{R}(t-1) \widetilde{r}(t-1), d \mathbf{Q}=\widetilde{R}(0) \cdots \widetilde{R}(T) d \mathbf{P}$. It follows that a.s.,

$$
\begin{aligned}
& E_{\mathbf{Q}}\left(\Delta S(t) \mid \mathcal{F}_{t-1}\right)=\tilde{R}(0) \cdots \tilde{R}(t-1) E\left(\Delta S(t) \tilde{R}(t) \cdots \tilde{R}(T) \mid \mathcal{F}_{t-1}\right) \\
& =\widetilde{R}(0) \cdots \widetilde{R}(t-1) E\left(\Delta S(t) \widetilde{R}(t) E\left(\widetilde{R}(t+1) \cdots \widetilde{R}(T) \mid \mathcal{F}_{t}\right) \mid \mathcal{F}_{t-1}\right) \\
& =\tilde{R}(0) \cdots \tilde{R}(t-1) E\left(\tilde{R}(t) \tilde{X}(t) \mid \mathcal{F}_{t-1}\right) \\
& \leq \widetilde{R}(0) \cdots \tilde{R}(t-1) \tilde{r}(t-1) S(t-1)=r(t-1) S(t-1),
\end{aligned}
$$

and similarly, $E_{\mathbf{Q}}\left((\Delta S(t)+D(t)) \mid \mathcal{F}_{t-1}\right) \geq r(t-1) S(t-1)$, showing (iii).

Finally, let us show that (iii) implies (i). Indeed, from (2.5), (2.6), and (3.2), we have a.s. that

$$
\begin{aligned}
E_{\mathbf{Q}}\left[V(t+1) \mid \mathcal{F}_{t}\right]= & V(t)+H^{+}(t+1) \cdot E_{\mathbf{Q}}\left[\Delta S(t+1) \mid \mathcal{F}_{t}\right] \\
& -H^{-}(t+1) \cdot E_{\mathbf{Q}}\left[\Delta S(t+1)+D(t+1) \mid \mathcal{F}_{t}\right]
\end{aligned}
$$




$$
\begin{aligned}
& \leq(1+r(t))\left(H^{+}(t+1)-H^{-}(t+1)\right) \cdot S(t) \\
& =(1+r(t)) V(t) .
\end{aligned}
$$

Suppose by contradiction that an arbitrage opportunity $H$ exists and (iii) holds. Let us denote by $V=(V(0), \ldots, V(T))$ the value process associated to the trading strategy $H$. Then, $V(0)=$ $0, V(T) \geq 0$, and $(V(T))>0$. We have now by backward induction in time that $V(t) \geq 0$ a.s. for all $t=T, \ldots, 0$. Indeed, from the definition of an arbitrage, the result is clearly true for $t=T$. Suppose $V(t) \geq 0$. From (B.3), it follows that $V(t) \geq 0$ (since $r(t) \geq 0$ ), showing the desired result. We show now by forward induction in time that, in fact, $V(t)=0$ a.s. for all $t=0, \ldots, T$. Indeed, for $t=0$, the result follows from the definition of an arbitrage. Suppose $V(t)=0$. From (B.3) and recalling that $V(t+1) \geq 0$ we get that $E_{\mathbf{Q}}\left[V(t+1) \mid \mathscr{F}_{t}\right]=0$. Taking the expected value, we obtain that $E_{\mathbf{Q}}[V(t+1)]=0$, which shows that $V(t+1)=0$ a.s., completing the induction argument. In particular, we have that $V(T)=0$, in contradiction with the fact that $E(V(T))>0$, showing the desired result.

Next, we present the proof of Corollary 3.4.

Proof. By backward induction on $t$, the result is true for $t=T$ by assumption. Suppose it holds for $t+1$, that is, a.s.

$$
E_{\mathbf{Q}}\left(\frac{X}{(1+r(T)) \cdots(1+r(t+1))} \mid \mathcal{F}_{t+1}\right) \leq V(t+1) .
$$

Then, from (B.3) and (B.4), we have a.s. that

$$
\begin{aligned}
& E_{\mathbf{Q}}(\left.\frac{X}{(1+r(T)) \cdots(1+r(t+1))} \mid \mathcal{F}_{t}\right) \frac{1}{1+r(t)} \\
& \quad= E_{\mathbf{Q}}\left(E_{\mathbf{Q}}\left(\frac{X}{(1+r(T)) \cdots(1+r(t+1))} \mid \mathcal{F}_{t+1}\right) \mid \mathcal{F}_{t}\right) \frac{1}{1+r(t)} \\
& \quad \leq E_{\mathbf{Q}}\left(V(t+1) \mid \mathcal{F}_{t}\right) \frac{1}{1+r(t)} \leq V(t)
\end{aligned}
$$

showing (3.4). We apply backward induction on $t$ to show that $\mathbf{P}(\Gamma(t))>0$. For $t=T$, the result is true by assumption. Suppose it holds for $t+1$. Thus we get that $\mathbf{Q}\left(\Gamma(t+1) \mid \mathfrak{F}_{t}\right)>0$ a.s. Therefore, we have from (B.3) that a.s.,

$$
\begin{aligned}
& E_{\mathbf{Q}}\left((1+r(t)) V(t)-\frac{X}{(1+r(T)) \cdots(1+r(t+1))} \mid \mathcal{F}_{t}\right) \\
& \quad \geq E_{\mathbf{Q}}\left(\left\{V(t+1)-E_{\mathbf{Q}}\left(\frac{X}{(1+r(T)) \cdots(1+r(t+1))} \mid \mathcal{F}_{t+1}\right)\right\} 1_{\Gamma(t+1)} \mid \mathcal{F}_{t}\right)>0,
\end{aligned}
$$

which implies, after dividing by $\left(1+r(t)\right.$ that $E_{\mathbf{Q}}\left(V(t)-X /(1+r(T)) \cdots(1+r(t)) \mid \mathcal{F}_{t}\right)>0$ a.s., yielding the desired result.

In what follows, we present the proof of Propositions 3.6 and 3.7. 
Proof. We take $r(t)=0$ and $\mathbf{Q} \in \Theta^{a}$ and show that $(\{r(t)\}, \mathbf{Q}) \in \mathcal{K}$. Indeed, recalling that $D(t+1) \geq 0$ we have a.s. that on $\left\{a_{i}(t)=0\right\}$ :

$$
E_{\mathbf{Q}}\left(\Delta S_{i}(t+1) \mid \mathscr{F}_{t}\right)=0 \leq E_{\mathbf{Q}}\left(D_{i}(t+1) \mid \mathscr{F}_{t}\right)=E_{\mathbf{Q}}\left(\Delta S_{i}(\mathrm{t}+1)+D_{i}(t+1) \mid \mathscr{F}_{t}\right)
$$

and similarly on $\left\{a_{i}(t)=1\right\}$,

$$
E_{\mathbf{Q}}\left(\Delta S_{i}(t+1)+D_{i}(t+1) \mid \mathscr{F}_{t}\right)=0 \geq E_{\mathbf{Q}}\left(\Delta S_{i}(t+1) \mid \mathcal{F}_{t}\right) .
$$

Proof. Consider $r(t)=0$ and $\mathbf{Q} \in \Theta^{a^{H}}$. As shown in Proposition 3.6, $(\{r(t)\}, \mathbf{Q}) \in \mathcal{K}$. From the hypothesis that $H_{i}^{+}(t) H_{i}^{-}(t)=0$ and (2.6), we have a.s. that

$$
\begin{aligned}
E_{\mathbf{Q}}\left(V^{H}(t) \mid \mathcal{F}_{t-1}\right)= & V^{H}(t-1)+\sum_{\left\{i \in\left\{a^{H}(t-1)=0\right\}\right\}} H_{i}^{+}(t) E_{\mathbf{Q}}\left(\Delta S_{i}(t) \mid \mathcal{F}_{t-1}\right) \\
& -\sum_{\left\{i \in\left\{a^{H}(t-1)=1\right\}\right\}} H_{i}^{-}(t) E_{\mathbf{Q}}\left(\Delta S_{i}(t)+D_{i}(t) \mid \mathcal{F}_{t-1}\right) \\
= & V^{H}(t-1),
\end{aligned}
$$

and thus a.s., $V^{H}(t)=E_{\mathbf{Q}}\left(X \mid \mathcal{F}_{t}\right)$ for all $t=0, \ldots, T-1$. From Corollary 3.4, we have the result.

\section{Acknowledgment}

The authors would like to express their gratitude to the referee for some helpful comments. O. L. V. Costa received financial support from CNPq (Brazilian National Research Council), Grant 301067/09-0, and USP Project MaCLinC.

\section{References}

[1] H. Markowitz, "Portfolio selection," The Journal of Finance, vol. 7, pp. 77-91, 1952.

[2] H. M. Markowitz, Portfolio Selection: Efficient Diversification of Investments, John Wiley \& Sons, New York, NY, USA, 1959.

[3] O. L. V. Costa and A. C. Paiva, "Robust portfolio selection using linear-matrix inequalities," Journal of Economic Dynamics E Control, vol. 26, no. 6, pp. 889-909, 2002.

[4] M. A. Howe and B. Rustem, "A robust hedging algorithm," Journal of Economic Dynamics \& Control, vol. 21, no. 6, pp. 1065-1092, 1997.

[5] M. A. Howe, B. Rustem, and M. J.P. Selby, "Multi-period minimax hedging strategies," European Journal of Operational Research, vol. 93, no. 1, pp. 185-204, 1996.

[6] R. Roll, "A mean/variance analysis of tracking error," The Journal of Portfolio Management, vol. 18, no. 4, pp. 13-22, 1992.

[7] B. Rustem, R. G. Becker, and W. Marty, "Robust min-max portfolio strategies for rival forecast and risk scenarios," Journal of Economic Dynamics \& Control, vol. 24, no. 11-12, pp. 1591-1621, 2000.

[8] M. C. Steinbach, "Markowitz revisited: mean-variance models in financial portfolio analysis," SIAM Review, vol. 43, no. 1, pp. 31-85, 2001.

[9] D. Li and W.-L. Ng, "Optimal dynamic portfolio selection: multiperiod mean-variance formulation," Mathematical Finance, vol. 10, no. 3, pp. 387-406, 2000. 
[10] N. Bäuerle and U. Rieder, "Portfolio optimization with Markov-modulated stock prices and interest rates," Institute of Electrical and Electronics Engineers. Transactions on Automatic Control, vol. 49, no. 3, pp. 442-447, 2004.

[11] U. Çakmak and S. Özekici, "Portfolio optimization in stochastic markets," Mathematical Methods of Operations Research, vol. 63, no. 1, pp. 151-168, 2006.

[12] O. L. V. Costa and M. V. Araujo, “A generalized multi-period mean-variance portfolio optimization with Markov switching parameters," Automatica, vol. 44, no. 10, pp. 2487-2497, 2008.

[13] O. L. V. Costa and W. L. de Paulo, "Indefinite quadratic with linear costs optimal control of Markov jump with multiplicative noise systems," Automatica, vol. 43, no. 4, pp. 587-597, 2007.

[14] O. L. V. Costa and R. B. Nabholz, "Multiperiod mean-variance optimization with intertemporal restrictions," Journal of Optimization Theory and Applications, vol. 134, no. 2, pp. 257-274, 2007.

[15] X. Y. Cui, D. Li, S. Y. Wang, and S. S. Zhu, "Better than dynamic mean-variance: time inconsistency and free cash flow stream," Mathematical Finance, vol. 22, no. 2, pp. 346-378, 2012.

[16] X. Li, X. Y. Zhou, and A. E. B. Lim, "Dynamic mean-variance portfolio selection with no-shorting constraints," SIAM Journal on Control and Optimization, vol. 40, no. 5, pp. 1540-1555, 2002.

[17] A. E. B. Lim and X. Y. Zhou, "Mean-variance portfolio selection with random parameters in a complete market," Mathematics of Operations Research, vol. 27, no. 1, pp. 101-120, 2002.

[18] S. O. Lozza, E. Angelelli, and A. Bianchi, "Financial applications of bivariate Markov processes," Mathematical Problems in Engineering, vol. 2011, Article ID 347604, 15 pages, 2011.

[19] G. Wang and Z. Wu, "Mean-variance hedging and forward-backward stochastic differential filtering equations," Abstract and Applied Analysis, vol. 2011, Article ID 310910, 20 pages, 2011.

[20] J. Xiong and X. Y. Zhou, "Mean-variance portfolio selection under partial information," SIAM Journal on Control and Optimization, vol. 46, no. 1, pp. 156-175, 2007.

[21] G. Yin and X. Y. Zhou, "Markowitz's mean-variance portfolio selection with regime switching: from discrete-time models to their continuous-time limits," Institute of Electrical and Electronics Engineers. Transactions on Automatic Control, vol. 49, no. 3, pp. 349-360, 2004.

[22] S. A. Zenios, Financial Optimization, Cambridge University Press, New York, NY, USA, 1993.

[23] Q. Zhang, "Stock trading: an optimal selling rule," SIAM Journal on Control and Optimization, vol. 40, no. 1, pp. 64-87, 2001.

[24] X. Y. Zhou and D. Li, "Continuous-time mean-variance portfolio selection: a stochastic LQ framework," Applied Mathematics and Optimization, vol. 42, no. 1, pp. 19-33, 2000.

[25] X. Y. Zhou and G. Yin, "Markowitz's mean-variance portfolio selection with regime switching: a continuous-time model," SIAM Journal on Control and Optimization, vol. 42, no. 4, pp. 1466-1482, 2003.

[26] F. Black and M. Scholes, "The pricing of options and corporate liabilities," Journal of Political Economy, vol. 81, no. 3, pp. 637-659, 1973.

[27] R. C. Merton, “Theory of rational option pricing," The Rand Journal of Economics, vol. 4, pp. 141-183, 1973.

[28] J. M. Harrison and D. M. Kreps, "Martingales and arbitrage in multiperiod securities markets," Journal of Economic Theory, vol. 20, no. 3, pp. 381-408, 1979.

[29] J. M. Harrison and S. R. Pliska, "Martingales and stochastic integrals in the theory of continuous trading," Stochastic Processes and their Applications, vol. 11, no. 3, pp. 215-260, 1981.

[30] R. C. Dalang, A. Morton, and W. Willinger, "Equivalent martingale measures and no-arbitrage in stochastic securities market models," Stochastics and Stochastics Reports, vol. 29, no. 2, pp. 185-201, 1990.

[31] N. Christopeit and M. Musiela, "On the existence and characterization of arbitrage-free measures in contingent claim valuation," Stochastic Analysis and Applications, vol. 12, no. 1, pp. 41-63, 1994.

[32] M. H. A. Davis, V. G. Panas, and T. Zariphopoulou, "European option pricing with transaction costs," SIAM Journal on Control and Optimization, vol. 31, no. 2, pp. 470-493, 1993.

[33] F. Delbaen and W. Schachermayer, "A general version of the fundamental theorem of asset pricing," Mathematische Annalen, vol. 300, no. 3, pp. 463-520, 1994.

[34] R. J. Elliott, L. Chan, and T. K. Siu, "Option pricing and Esscher transform under regime switching," Annals of Finance, vol. 1, no. 4, pp. 423-432, 2005.

[35] R. J. Elliott, T. K. Siu, L. Chan, and J. W. Lau, "Pricing options under a generalized Markov-modulated jump-diffusion model," Stochastic Analysis and Applications, vol. 25, no. 4, pp. 821-843, 2007.

[36] Y. M. Kabanov, "Hedging and liquidation under transaction costs in current markets," Finance and Stochastics, vol. 3, pp. 237-248, 1999. 
[37] A. V. Melnikov and Y. G. Petrachenko, "On option pricing in binomial market with transaction costs," Finance and Stochastics, vol. 9, no. 1, pp. 141-149, 2005.

[38] L. Stettner, "Option pricing in discrete-time incomplete market models," Mathematical Finance, vol. 10, no. 2, pp. 305-321, 2000.

[39] P. P. Boyle and T. Vorst, "Option replication in discrete time with transactions costs," The Journal of Finance, vol. 47, no. 1, pp. 271-293, 1992.

[40] J. C. Cox, S. A. Ross, and M. Rubinstein, "Option pricing: a simplified approach," Journal of Financial Economics, vol. 7, no. 3, pp. 229-263, 1979.

[41] J. Cvitanic, "Minimizing expected loss of hedging in incomplete and constrained markets," SIAM Journal on Control and Optimization, vol. 38, no. 4, pp. 1050-1066, 2000.

[42] C. Edirisinghe, V. Naik, and R. Uppal, "Optimal replication of options with transactions costs and trading restrictions," Journal of Financial and Quantitative Analysis, vol. 28, no. 1, pp. 117-138, 1993.

[43] H. E. Leland, "Option pricing and replication with transactions costs," The Journal of Finance, vol. 40, no. 5, pp. 1283-1301, 1985.

[44] H. Liu, "Optimal consumption and investment with transaction costs and multiple risky assets," Journal of Finance, vol. 59, no. 1, pp. 289-338, 2004.

[45] E. Jouini and H. Kallal, "Arbitrage in securities markets with short-sales constraints," Mathematical Finance, vol. 5, no. 3, pp. 197-232, 1995.

[46] H. Pham and N. Touzi, "The fundamental theorem of asset pricing with cone constraints," Journal of Mathematical Economics, vol. 31, no. 2, pp. 265-279, 1999.

[47] C. Napp, "The Dalang-Morton-Willinger theorem under cone constraints," Journal of Mathematical Economics, vol. 39, no. 1-2, pp. 111-126, 2003.

[48] Yu. M. Kabanov and D. O. Kramkov, "Nonarbitrage and equivalent martingale measures: a new proof of the Harrison-Pliska theorem," Probability Theory and Applications, vol. 39, no. 3, pp. 635-640, 1994.

[49] W. Schachermayer, "A Hilbert space proof of the fundamental theorem of asset pricing in finite discrete time," Insurance: Mathematics E Economics, vol. 11, no. 4, pp. 249-257, 1992.

[50] R. J. Elliot and P. E. Kopp, Mathematics of Finance Markets, Springer Verlag, Berlin, Germany, 2004.

[51] S. R. Pliska, Introduction to Mathematical Finance, Blackwell Publishing, Oxford, Uk, 1997.

[52] Y. Kabanov and C. Stricker, "A teachers' note on no-arbitrage criteria," in Séminaire de Probabilités, XXXV, vol. 1755 of Lecture Notes in Mathematics, pp. 149-152, Springer, Berlin, Germany, 2001. 


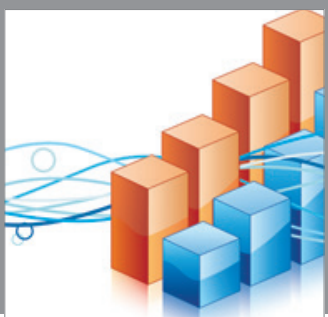

Advances in

Operations Research

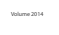

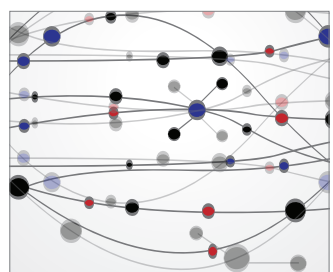

\section{The Scientific} World Journal
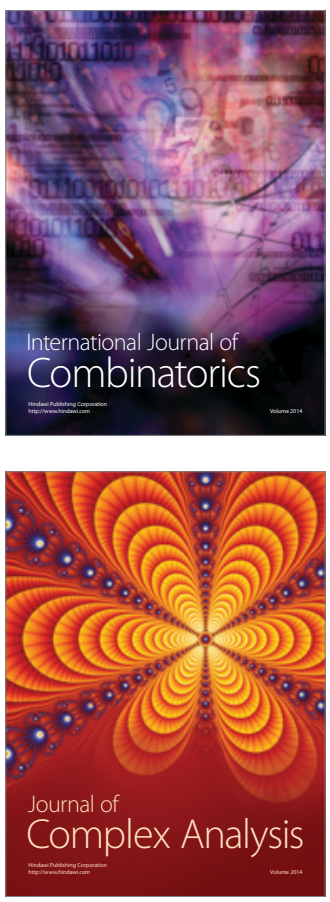

International Journal of

Mathematics and

Mathematical

Sciences
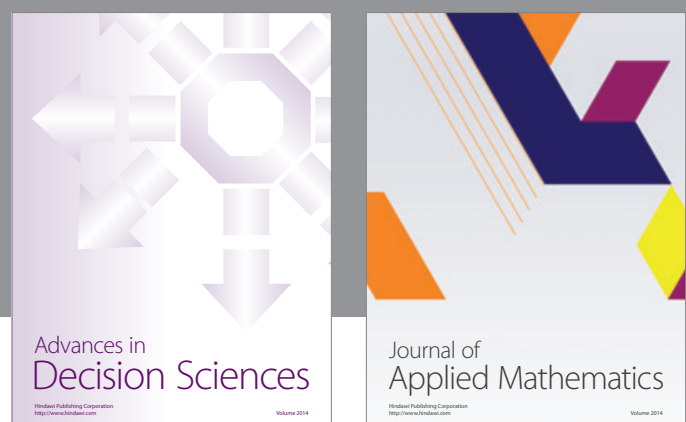

Journal of

Applied Mathematics
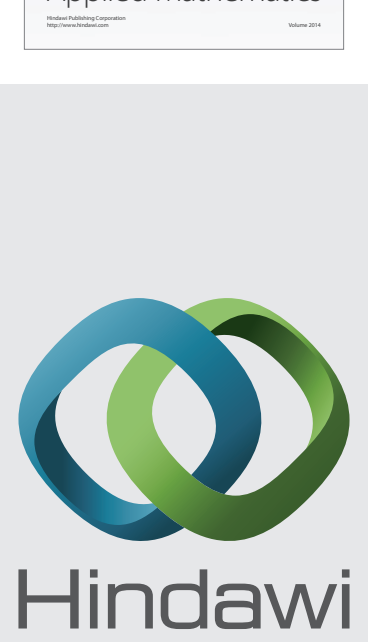

Submit your manuscripts at http://www.hindawi.com
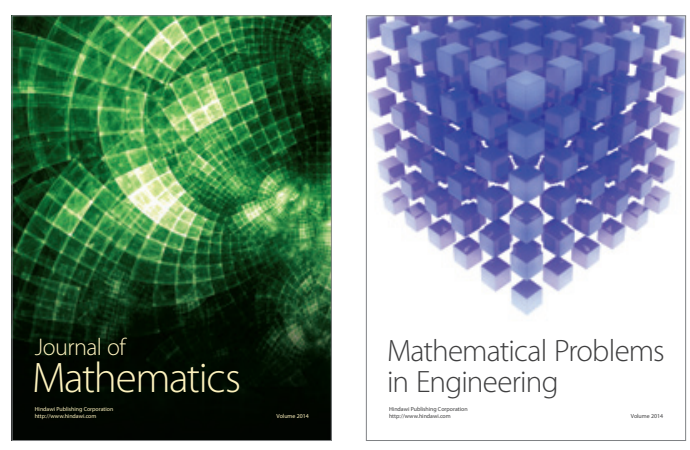

Mathematical Problems in Engineering
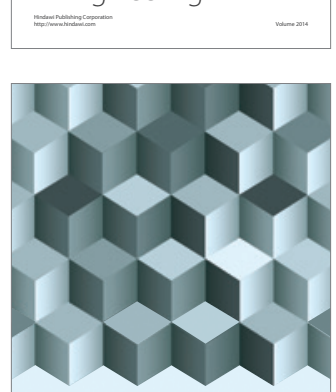

Journal of

Function Spaces
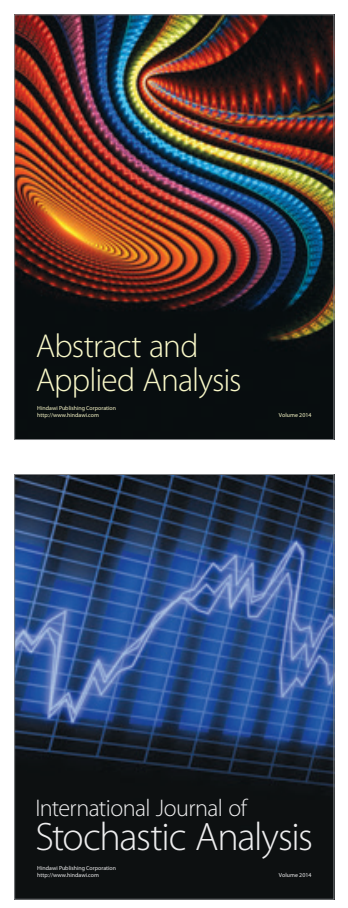

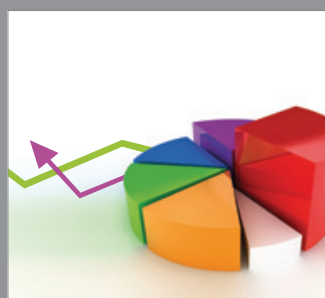

ournal of

Probability and Statistics

Promensencen
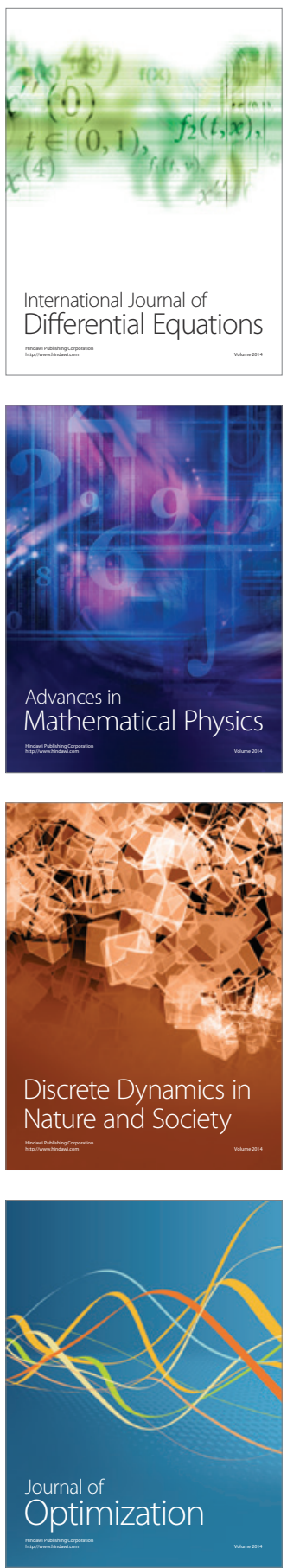